\title{
Point-of-care ultrasound: reply to Andronikou et al. and Györgyi et al.
}

\author{
Owen J. Arthurs ${ }^{1,2,3}$ (D) $\cdot$ Rick R. van Rijn $^{4} \cdot$ Samuel Stafrace ${ }^{5,6} \cdot$ Karen Rosendahl ${ }^{7,8}$ \\ Received: 3 August 2021 / Revised: 3 August 2021 / Accepted: 11 August 2021 / Published online: 24 September 2021 \\ (C) The Author(s), under exclusive licence to Springer-Verlag GmbH Germany, part of Springer Nature 2021
}

Dear Editors,

We read with interest the letters of Andronikou et al. [1] and Györgyi et al. [2] regarding the European Society of Paediatric Radiology (ESPR) position statement on non-radiologist point-of-care ultrasound (POCUS) [3].

We are delighted that our article [3] and associated reviews of practice [4] are generating discussion and debate amongst the wider paediatric radiology community. This discussion is welcome because it demonstrates both the importance of this topic and the wide range of circumstances in which nonradiologist POCUS is being practised or considered.

We overwhelmingly agree with the main point made in both recent letters to this journal regarding the major impact to the patient in the face of a limited workforce that nonradiologist POCUS can offer: rapid access to US services in an underserved population, particularly important in the developing world. The ESPR has an active outreach educational program with a specific outreach taskforce [5] in collaboration with the World Federation of Pediatric Imaging (WFPI), amongst others.

Owen J. Arthurs

Owen.arthurs@gosh.nhs.uk

1 Great Ormond Street Hospital for Children NHS Foundation Trust, London WC1N 3JH, UK

2 UCL Great Ormond Street Institute of Child Health, London, UK

3 NIHR Great Ormond Street Hospital Biomedical Research Centre, London, UK

4 Department of Radiology, Emma Children's Hospital-Amsterdam UMC, University of Amsterdam, Amsterdam, The Netherlands

5 Department of Diagnostic Imaging, Sidra Medicine, Doha, Qatar

6 Weill Cornell Medicine, Doha, Qatar

7 Department of Radiology, University Hospital of North Norway, Tromsø, Norway

8 The Arctic University of Norway, Tromsø, Norway
Both Györgyi et al. [2] and Andronikou et al. [1] agreed with our position that a wide range of trained users are currently offering POCUS. We also recognise that much of the literature they cited on the effectiveness of non-radiologist POCUS recognises improvements in workload efficiency, reducing delays, potential cost efficiencies and consequences for overall health care costs [1].

No one can be surprised that making something more widely available (a) is feasible [6], (b) improves access and (c) elicits more diagnoses. We all recognise that placing a US probe on a child and generating images is highly appealing to both practitioner and patient but does not define a diagnostic US examination.

We encourage practitioners not to confuse availability with diagnostic accuracy. In their case study of lung US, only one of the papers put forwards by Andronikou et al. [1] discussed the diagnostic accuracy of POCUS [7]. In that study, 12 of 34 people with coronavirus disease 2019 (COVID-19), the disease caused by severe acute respiratory syndrome coronavirus 2 (SARS-CoV-2), had CT performed to compare against lung US. Despite overall agreement (normal/abnormal), each patient had discrepancies between CT and US findings. These are classic examples of type II error rates: if we search for agreement, we will not find discrepancies. Furthermore, whilst value judgements are by nature subjective, we must be careful not to conclude usefulness from observational studies when "usefulness" has neither been defined nor shown.

Andronikou et al. [1] also recognised that it is the limited experience rather than status of US practitioners that can generate errors. We could not agree more: clearly many radiologists make errors, but they do so in a regulated environment that highlights rather than hides errors. Part of the reason that radiologic US scans are documented, saved and formally reported in the clinical notes is to create an auditable trail, and errors are highlighted for future learning, e.g., Royal College of Radiologists' "Radiologists Events and Learning Meetings" standards [8]. Practicing outside of an 
effective governance structure should be questioned in the modern era of medical scrutiny and indemnity.

We collectively agree that US examinations should be performed by trained personnel and that high-quality reporting should be performed in a structured governance environment that is transparent, auditable and leads to further learning. We stand by our original position that all imaging findings should be documented, reproducible and open to external scrutiny, and our objective is to encourage all practitioners to meet these standards rather than to discredit those outside radiology who perform US.

It is naïve to think that any medical practitioner can obtain a US machine and begin making accurate diagnoses. To quote a Pediatric Radiology editor, "Ultrasound, regardless of where it is performed and by whom, requires training, practice and expertise to be clinically helpful; and its precision, diagnostic observations, interpretations and clinical efficacy are global" [9]. Putting the probe on the patient is the beginning, but far from the end, of a good US exam. We actively encourage all practitioners to come together to ensure children receive the best possible care rather than the care that is currently available.

\section{Declarations}

Conflicts of interest None

\section{References}

1. Andronikou S, Otero H, Belard S et al (2021) Radiologists should support non-radiologist point-of-care ultrasonography in children: a case for involvement and collaboration. Pediatr Radiol. https://doi. org/10.1007/s00247-021-05185-7

2. Györgyi Z, De Luca D, Singh Y (2021) The European Society of Paediatric Radiology's position statement on point-of-care ultrasound. Pediatr Radiol. https://doi.org/10.1007/s00247-021-05184-8

3. van Rijn RR, Stafrace S, Arthurs OJ et al (2021) Non-radiologistperformed point-of-care ultrasonography in paediatrics - European Society of Paediatric Radiology position paper. Pediatr Radiol 51: $161-167$

4. van Wassenaer EA, Daams JG, Benninga MA et al (2021) Nonradiologist-performed abdominal point-of-care ultrasonography in paediatrics - a scoping review. Pediatr Radiol 51:1386-1399

5. European Society of Paediatric Radiology (2021) Outreach. https:// www.espr.org/taskforces/outreach/. Accessed 9 Aug 2021

6. Conlon TW, Himebauch AS, Fitzgerald JC et al (2015) Implementation of a pediatric critical care focused bedside ultrasound training program in a large academic PICU. Pediatr Crit Care Med 16:219-226

7. Giorno EPC, De Paulis M, Sameshima YT et al (2020) Point-of-care lung ultrasound imaging in pediatric COVID-19. Ultrasound J 12:50

8. The Royal College of Radiologists (2020) Standards for radiology events and learning meetings. https://www.rcr.ac.uk/publication/ standards-radiology-events-and-learning-meetings. Accessed 28 Jul 2021

9. Olsen ØE (2017) Reply to Andronikou and Sergot: "point-of-care" ultrasound. Pediatr Radiol 47:1851-1852

Publisher's note Springer Nature remains neutral with regard to jurisdictional claims in published maps and institutional affiliations. 